\title{
Revisão da produção científica sobre a participação social na gestão ambiental municipal
}

\begin{abstract}
A participação social trata das tentativas de inclusão da sociedade nas políticas públicas, buscando atender um anseio da própria população e setores representativos por integrar os processos de tomadas de decisão, levando diretamente ao governo suas demandas nas mais diversas áreas que lhe dizem respeito e estão relacionadas ao bem comum, como a questão ambiental. Nesse contexto, a presente pesquisa teve por objetivo revisar sistematicamente a literatura sobre a participação social na gestão ambiental municipal. Através de busca sistêmica do tema nos periódicos indexados na base Web Of Science - WOS e do uso do software VOSViewer, foi possível analisar os dados bibliográficos de 56 artigos publicados no período de 2008 a 2018, selecionados por tratarem de algum aspecto da participação social na gestão ambiental municipal. Verificou-se um crescimento relativo das pesquisas nessa temática, apresentando um crescimento elevado no ano de 2017, e que essas pesquisas têm se concentrado na avaliação e desenvolvimento dessa participação em questões e áreas temáticas, como: quanto à problemática dos resíduos sólidos, a questão dos recursos hídricos, da gestão de áreas protegidas, do desenvolvimento da gestão ambiental e a sociedade, e uma pequena presença quanto às mudanças climáticas e energias renováveis. O tema que mais cresceu dentro das produções científicas analisadas, foi em relação à participação social voltada para a problemática ambiental dos resíduos sólidos a nível mundial. A investigação da produção científica revelou um campo de estudo em relativo crescimento, a partir de 2015; porém, ainda de forma fragmentada e sem redes de coautoria entre instituições e países. Parece haver um consenso sobre a importância do papel da participação social na gestão ambiental municipal; entretanto, apresentam-se poucos estudos. Em pesquisas futuras podem ser ampliadas as investigações para outros bancos de dados, como Scopus.
\end{abstract}

Palavras-chave: Meio ambiente; Sociedade; Participação.

\section{Review of scientific production on social participation in municipal environmental management}

\begin{abstract}
Social participation deals with the attempts to include society in public policies, seeking to meet a desire of the population itself and representative sectors to integrate decision-making processes, leading directly to the government their demands in various areas that concern and are related to it. to the common good such as the environmental issue. In this context, this research aimed to systematically review the literature on social participation in municipal environmental management. Through a systemic search of the theme in journals indexed in the Web of Science - WOS database and the use of VosViewer software, it was possible to analyze the bibliographic data of 56 articles published from 2008 to 2018, selected because they deal with some aspect of social participation in the journal. municipal environmental management. There was a relative growth of research on this topic, showing a high growth in 2017, and that this research has focused on the evaluation and development of this participation in issues and thematic areas, such as: regarding the solid waste problem, the issue water resources, protected area management, environmental management development and society, and a small presence on climate change and renewable energy. The theme that grew the most in the analyzed scientific productions was in relation to social participation focused on the environmental problem of solid waste worldwide. Research into scientific production has revealed a growing field of study from 2015; however, still in a fragmented way and without co-authoring networks between institutions and countries. There seems to be consensus on the importance of the role of social participation in municipal environmental management; However, few studies are presented. Future research may extend investigations to other databases such as Scopus.
\end{abstract}

Keywords: Environment; Society; Participation.

Topic: Planejamento, Gestão e Políticas Públicas Ambientais

Reviewed anonymously in the process of blind peer.
Received: 28/06/2019

Approved: 29/07/2019
Maria das Dores de Souza Abreu Alencar id

Universidade Federal de Campina Grande, Brasil http://lattes.cnpq.br/2823539466395889

http://orcid.org/0000-0001-7794-3144

mdabreu.bio@gmail.com

Lúcia Santana de Freitas (it)

Universidade Federal de Campina Grande, Brasil

http://lattes.cnpq.br/4689902016970348

http://orcid.org/0000-0001-8185-0151

lucia.sdefreitas@gmail.com
Referencing this:

ALENCAR, M. D. S. A.; FREITAS, L. S.. Revisão da produção científica sobre a participação social na gestão ambiental municipal. Revista Ibero-Americana de Ciências Ambientais, v.10, n.4, p.345-358, 2019. DOI: http://doi.org/10.6008/CBPC2179-6858.2019.004.0027 


\section{INTRODUÇÃO}

A necessidade de entendimento da relação entre homem e natureza considerando a força do capitalismo e da visão utilitarista sobre os recursos naturais tem perpassado gerações, levando ao surgimento de legislações e ações direcionadas para a gestão adequada do uso dos recursos naturais para manutenção e continuidade da sociedade e controle da pressão que esses usos exercem sobre o meio ambiente e que podem comprometer a existência da própria sociedade e de sua qualidade de vida.

Nesse contexto, a gestão ambiental vem se desenvolvendo nas últimas décadas, como resultado da necessidade de adequação a uma nova forma de pensar em desenvolvimento e produção de bens de consumo, circunscrita pelo desenvolvimento sustentável (MEDEIROS et al., 2012), podendo ser definida como um conjunto de ações envolvendo políticas públicas, setor produtivo e sociedade civil, para garantir a sustentabilidade dos recursos ambientais, da qualidade de vida e do próprio processo de desenvolvimento, dentro de um complexo sistema de interações da humanidade com os ecossistemas (BURSZTYN et al., 2012).

No que tange a gestão ambiental pública, considera-se essencialmente ser uma gestão de conflitos, implicando, portanto, na construção de mecanismos e ferramentas políticas capazes de atuarem com eficiência em um mundo em processo acelerado de mudanças. Em vista disso, fazer gestão ambiental pública é uma tarefa complexa.

Nessa perspectiva, os municípios brasileiros passaram a ser reconhecidos como entes federativos autônomos para as práticas de proteção dos recursos naturais a partir da Constituição Brasileira de 1988, a qual consolidou em solo nacional a questão da preservação do meio ambiente (BRASIL, 1988). Na atualidade, existe cada vez mais a necessidade de os municípios tornarem-se protagonistas da sustentabilidade do seu desenvolvimento, exigindo nesse contexto que as administrações municipais se estruturem e capacitaremse para cumprir o dever de gerenciar as questões ambientais da sua localidade, através da execução de instrumentos de gestão ambiental. Após a Constituição de 1988, o papel dos governos locais vem sendo acrescido, saindo da visão de meros prestadores de serviços para o de agentes de desenvolvimento local (ÁVILA, 2012).

Dentre os desafios e obstáculos para o desenvolvimento da gestão ambiental municipal estão: a baixa escolaridade e capacidade técnica dos servidores desses setores e assim ausência de capital humano, recursos limitados para investimentos nessa área, ausência de prioridade política, ausência de informação por parte dos gestores e da população, e a inexistência ou insuficiência de participação social dos munícipes e demais instituições participantes, sendo essa uma das áreas atuais de relevante interesse da gestão ambiental que demandam investimentos e pesquisas que visem o desenvolvimento e fortalecimento da efetividade da gestão ambiental municipal através do envolvimento da participação social (SIQUEIRA, 2008; FARIAS, 2011; LOPEZ, 2014).

Os estudos sobre participação social permeiam diversas áreas do conhecimento, como sociologia, direito, ciências políticas, psicologia e administração, entre outras, sendo, portanto, tratada com diferentes nomenclaturas como inclusão social, participação cidadã, participação popular, participação democrática, 
entre outras, que independente da área em questão trata das tentativas de inclusão da sociedade nas políticas públicas, buscando, segundo Simões et al. (2015), atender um anseio da própria população por integrar processos de tomadas de decisão levando diretamente ao governo suas demandas nas mais diversas áreas que lhe dizem respeito e estão relacionadas ao bem comum, como a questão ambiental.

A participação social é uma consolidação do processo democrático no fortalecimento da cidadania, e diz respeito à construção de espaços que criam interconexões entre os gestores e a sociedade (CARVALHO, 1998). Sendo uma conquista da sociedade, significa uma busca pela democratização, e tem significado histórico para países que viveram em regimes autoritários, pela luta a participação tornou-se um direito do cidadão (GARBELINE, 2017). A participação social em matéria ambiental é um princípio constitucional e expressa a ideia de que para a resolução dos problemas ambientais, a cooperação entre o Estado e a sociedade se dá com participação dos diferentes grupos sociais na formulação e na execução da política ambiental, nas esferas federal, estadual e municipal (PEREIRA, 2015).

No contexto municipal, um Sistema Municipal do Meio Ambiente - SISMUMA organizado e atuante decorre de um processo fortemente vinculado à participação da sociedade local e às características de sua realidade socioambiental. A participação não deve se restringir às fases de formulação e criação desse sistema. Destacando que o cotidiano da gestão ambiental municipal também requer participação qualificada. A população pode contribuir na identificação e na solução dos problemas. Desde que haja vontade política e sejam criados os mecanismos para isso, a participação social pode promover a responsabilidade coletiva e um efetivo controle social sobre as ações da gestão ambiental pública. Quando as pessoas se comprometem com alguma ação ambiental, tornam-se corresponsáveis pelos resultados e mais capazes de manter esse compromisso ao longo do tempo, tornando assim as ações mais permanentes.

Nesse sentido, conhecer como essa participação social na gestão ambiental municipal vem sendo tratada na literatura, tais como: atores envolvidos, espaços em que ocorrem, dificuldades, limitações e desafios, estratégias e instrumentos utilizados, mecanismos de envolvimento e entre outros, é de fundamental importância para o avanço dessa temática. Diante do exposto o presente trabalho tem como objetivo revisar sistematicamente a literatura sobre a participação social na gestão ambiental municipal, publicada no período de 2008 a 2018.

\section{METODOLOGIA}

Trata-se de um estudo baseado na avaliação da produção científica sobre a participação social na gestão ambiental municipal, mediante a análise de dados bibliográficos, bem como da discussão sobre as redes de coautoria, análise de texto, temáticas abordadas e o avanço da produção nessa área ao longo do período de 2008 a 2018, entre outros aspectos possíveis de analisar. Para tanto, foram utilizadas as bases de dados disponíveis na Web Of Science - WOS, sendo elas: a Principal Coleção da WOS que traz os principais periódicos acadêmicos e anais nas ciências, ciências sociais, artes e humanas, a DIIDW-Derwent Innovations Index, KJD-KCl - Base de dados de periódicos coreanos, a RSCl - Russian Science Citation Index e o SCIELO (SciELO Citation Index) que fornece literatura acadêmica em ciências, ciências sociais, artes e humanas 
publicada nos principais periódicos de acesso aberto da América Latina, Portugal, Espanha e África do Sul.

Quanto à investigação na base de dados Web Of Science (WOS), considerou-se os artigos publicados em todas as bases de dados sobre o assunto investigado 'Municipal Enviromental Managenet' and 'Social Participation' no período de 2008 a 2018. Foram estipulados critérios para a busca sistêmica do tema nos periódicos indexados na base WOS conforme pode se observar pela figura 1.

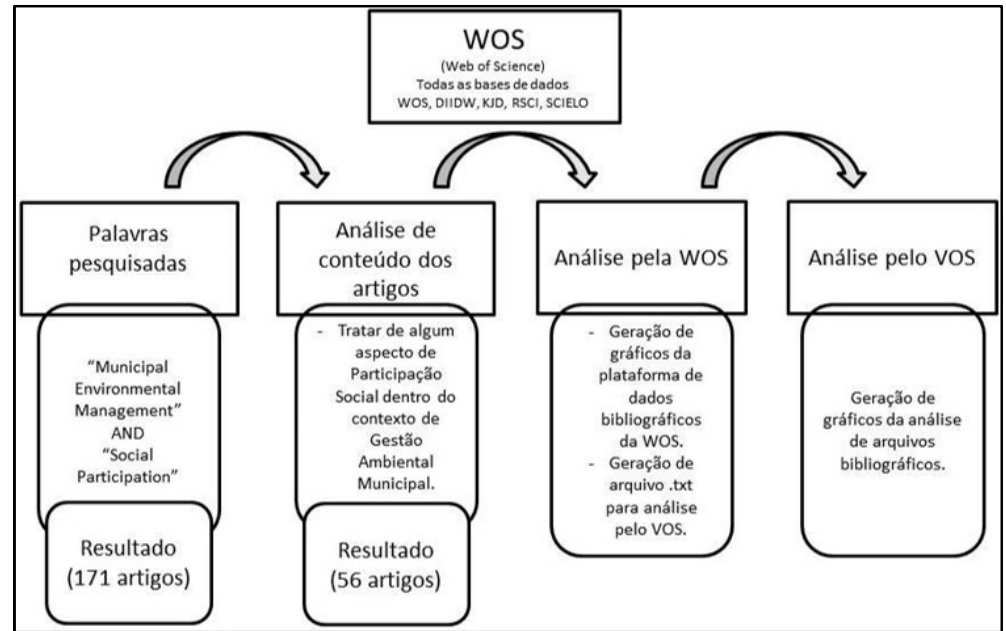

Figura 1: Delineamento para busca sistêmica na Web Of Science - WOS

Através do uso dos descritores 'Municipal Envirolmental Managenet' e 'Social Participation' em pesquisa realizada em março de 2019 na plataforma dos periódicos da Capes na base dados da Wef Of Science (WOS) considerando a opção para todas as bases de dados, utilizando na busca avançada os critérios de busca, como: a combinação: TS=(Municipal Environmental Management AND Social Participation), presentes no título, resumo e palavras-chaves dos artigos, em todos os idiomas e publicados em periódicos no período de 2008 a 2018, foram encontrados 171 artigos científicos.

Em seguida foi feita a leitura do resumo e considerações finais dos 171 artigos, onde foram selecionados artigos que tratassem de algum aspecto da participação social na gestão ambiental municipal. Foram selecionados 56 artigos que foram compor uma lista marcada criada na base da WOS para análise mais detalhada posteriormente. Na ocasião foi gerado também um arquivo em formato (.txt) necessário para utilização para o tratamento dos dados, utilizando o VOSViewer ${ }^{\circledR}$, que é um software de visualização de similaridades 'visualization of similiarities' - VOS, em sua versão 1.6.11, que foca na visualização e construção de redes.

O VOSviewer é um software projetado especificamente para construir e visualizar mapas do conhecimento científico a partir de informações bibliográficas. A ferramenta foi desenvolvida pelo Centro de Ciência e Tecnologia Estudos da Universidade de Leiden, na Holanda. Entretanto, embora o VOSviewer possa ser usado para construir e visualizar mapas de qualquer tipo de dados de co-ocorrência não tem capacidades de pré-processamento, o que significa que é necessário usar software externo para preparar os dados para análise e, em seguida, representação (LILLO et al., 2017). 


\section{RESULTADOS E DISCUSSÃO}

\section{Evolução temporal da produção}

O desenvolvimento da produção científica entre os anos de 2008 e 2014 a quantidade de artigos variou entre 1 a 4 no máximo, e a partir de 2015 elevou-se, tendo variado entre 6 e 16 nos anos de 2015 a 2018, como pode ser visto no Gráfico1. Com base na análise do gráfico 1, observa-se um aumento das pesquisas em participação social na gestão ambiental municipal ao longo dos anos. Verifica-se, portanto, que entre 2008 a 2014 houve oscilações entre crescimento e decrescimento, somente a partir de 2015 apresenta só crescimento, sendo mais significativo no ano de 2017.

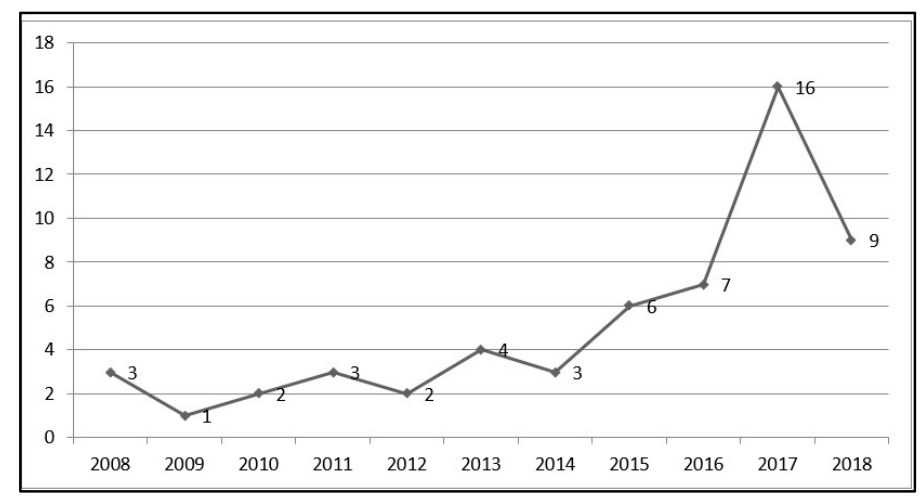

Gráfico 1: Crescimento da pesquisa científica em participação social na gestão ambiental municipal

\section{Periódicos com mais publicações}

Quanto às revistas e espaços de publicação encontrados aparecem em primeiro lugar, como pode ser visto na Tabela 3 abaixo, o Journal of Cleaner Production e o Resources e o Conservation and Recycling, com 5 artigos publicados, os dois são revistas internacionais, transdisciplinares, voltadas para a pesquisa e a prática de Produção Mais Limpa, Ambiental e Sustentável, considerando o manejo sustentável e a conservação de recursos, enfatizando os processos de transformação envolvidos em uma transição para sistemas de produção e consumo mais sustentáveis.

Tabela 3: Periódicos com publicações sobre Participação Social na Gestão Ambiental Municipal.

\begin{tabular}{|c|c|}
\hline Periódico & Quantidade de Artigos \\
\hline Journal of Cleaner Production & 5 \\
\hline Resources e o Conservation and Recycling & 5 \\
\hline Journal of Material Cycles and Waste Management & 3 \\
\hline Waste Management & 3 \\
\hline Environmental Policy and Governance & 2 \\
\hline Environmental Science Policy & 2 \\
\hline Journal of Envrionmental Management & 2 \\
\hline Waste Management Research & 2 \\
\hline Demais Periódicos (total de 32) & 1 \\
\hline Total & $\mathbf{5 6}$ \\
\hline
\end{tabular}

Em segundo lugar aparecem o Journal of Material Cycles and Waste Management e o Waste Management com 3 artigos publicados em cada um. Em terceiro, estão o Environmental Policy and Governance; Environmental Science Policy; Journal of Envrionmental Management e o Waste Management Research com 2 artigos em cada. E os demais periódicos num total de 32 tiveram apenas 1 artigo publicado cada um. 
Os artigos com maior número de citações, considerando que as citações estão relacionadas com o impacto do artigo, o que se torna relevante para a (re)construção do conhecimento científico; os autores mais citados para a produção científica no campo do conhecimento científico, bem como os periódicos com os seus respectivos artigos, que mais foram citados, podem ser visto na tabela 4 abaixo.

Tabela 4: Artigos com maior número de citações.

\begin{tabular}{|c|c|c|c|}
\hline Artigo & Título & Periódico & Citações \\
\hline $\begin{array}{l}\text { Agamuthu et al. } \\
\text { (2011) }\end{array}$ & $\begin{array}{l}\text { Challenges and issues in moving towards sustainable landfilling in a transitory } \\
\text { country: Malaysia. }\end{array}$ & $\begin{array}{l}\text { Waste Management \& } \\
\text { Research }\end{array}$ & 37 \\
\hline Gutberlet (2008) & $\begin{array}{l}\text { Empowering collective recycling initiatives: Video documentation and action } \\
\text { research with a recycling co-op in Brazil. }\end{array}$ & $\begin{array}{l}\text { Resources, Conservation and } \\
\text { Recycling }\end{array}$ & 30 \\
\hline Ma et al. (2016) & $\begin{array}{l}\text { Exploring social dimensions of municipal solid waste management around the } \\
\text { globe: A systematic literature review }\end{array}$ & Waste Management & 23 \\
\hline $\begin{array}{l}\text { Zhang et al. } \\
(2016)\end{array}$ & $\begin{array}{l}\text { What keeps Chinese from recycling: Accessibility of recyclingfacilities and the } \\
\text { behavior. }\end{array}$ & $\begin{array}{l}\text { Resources, Conservation and } \\
\text { Recycling }\end{array}$ & 20 \\
\hline Che et al. (2013) & $\begin{array}{l}\text { Residents' concerns and attitudes toward a municipal solid waste landfill: } \\
\text { integrating a questionnaire survey and GIS techniques. }\end{array}$ & $\begin{array}{l}\text { Environmental Monitoring } \\
\text { and Assessment }\end{array}$ & 19 \\
\hline $\begin{array}{l}\text { Soma et al. } \\
(2010)\end{array}$ & $\begin{array}{l}\text { Is There Anything Like a Citizen? A Descriptive Analysis of Instituting a } \\
\text { Citizen's Role to Represent Social Values at the Municipal Level }\end{array}$ & $\begin{array}{l}\text { Environmental Policy and } \\
\text { Governance }\end{array}$ & 19 \\
\hline $\begin{array}{l}\text { Fleeger et al. } \\
(2008)\end{array}$ & $\begin{array}{l}\text { Creating and sustaining community capacity for ecosystem-based } \\
\text { management: Is local government the key? }\end{array}$ & $\begin{array}{l}\text { Journal of Environmental } \\
\text { Management }\end{array}$ & 18 \\
\hline $\begin{array}{l}\text { Triguero et al. } \\
(2016)\end{array}$ & $\begin{array}{l}\text { Factors influencing willingness to accept different waste management } \\
\text { policies: empirical evidence from the European Union }\end{array}$ & Journal of Cleaner Production & 16 \\
\hline $\begin{array}{l}\text { Bergsma et al. } \\
(2012)\end{array}$ & $\begin{array}{l}\text { Does individual responsibility increase the adaptive capacity of society? The } \\
\text { case of local water management in the Netherlands }\end{array}$ & $\begin{array}{l}\text { Resources, Conservation and } \\
\text { Recycling }\end{array}$ & 16 \\
\hline $\begin{array}{l}\text { Soma et al. } \\
(2010)\end{array}$ & $\begin{array}{l}\text { Framing Participation with Multicriterion Evaluations to Support the } \\
\text { Management of Complex Environmental Issues }\end{array}$ & $\begin{array}{l}\text { Environmental Policy and } \\
\text { Governance }\end{array}$ & 15 \\
\hline
\end{tabular}

Pode-se observar que 4 dos 10 artigos mais citados apresentam entre 20 e 37 citações, e 6 deles apresentam entre 15 e 19, e que $90 \%$ dos artigos mais citados foram publicados em periódicos que aparecem na Tabela 3 anterior como os que apresentam entre 2 e 5 artigos publicados, e somente 1 dos artigos mais citados foi publicado em um periódico que apresenta somente 1 artigo publicado.

Dentre os autores mais citados, apontam-se: Agamuthu et al. (2011) e Gutberlet (2008), com seus trabalhos sobre a participação social na gestão de resíduos sólidos em âmbito local, publicados em periódicos direcionados para a pesquisa e a prática de Produção Mais Limpa, Ambiental e Sustentável, considerando o manejo sustentável e a conservação de recursos, enfatizando os processos de transformação envolvidos em uma transição para sistemas de produção e consumo mais sustentáveis, o que evidencia o avanço e continuidade dos estudos direcionados à busca por soluções para a problemática ambiental do gerenciamento dos resíduos sólidos, trazendo em suas abordagens a participação social dentro desse contexto.

\section{Instituições de origem dos artigos}

Analisando a afiliação dos autores em relação às instituições de origem de atuação, pode-se observar a Tabela 5 abaixo, 10 instituições apresentam uma quantidade de 2 artigos cada uma com autores afiliados, e assim outras 36 instituições com apenas 1 artigo publicado por autores afiliados. Pode-se identificar uma produção bastante dispersa entre os países, considerando que entre os países de origem das instituições dos autores que apresentam pelo menos 2 artigos publicados, a China aparece em primeiro lugar com 4 instituições das 10 mais presentes. Em segundo lugar aparece o Brasil e a Noruega com duas instituições e em terceiro a Austrália e o Canadá com uma instituição como origem dos autores de pelo menos dois artigos 
publicados.

Tabela 5: Filiação dos pesquisadores.

\begin{tabular}{|l|c|c|}
\hline Instituições & Quantidade de Artigos & País \\
\hline Agricultural Economics Research Institute & 2 & Noruega \\
\hline Australian National University & 2 & Austrália \\
\hline Chinese Academy of Science & 2 & China \\
\hline Hong Kong Polytechnic University & 2 & China \\
\hline Institute of Urban Environment & 2 & China \\
\hline Norwegian University of Life Science & 2 & Noruega \\
\hline Universidade Federal de Minas Gerais & 2 & Brasil \\
\hline Universidade Federal do Pará & 2 & Brasil \\
\hline University of Waterloo & 2 & Canadá \\
\hline Zhegiang University & 2 & China \\
\hline Demais instituições (total de 36) & 1 & Variados \\
\hline Total & $\mathbf{5 6}$ & \\
\hline
\end{tabular}

\section{Autores mais produtivos e redes de coautoria}

Os principais autores com mais artigos publicados sobre Participação Social na Gestão Ambiental Municipal (Ver tabela 2). Verifica-se que apenas quatro autores publicaram pelo menos dois artigos sobre essa temática, sendo eles Katrine Soma da Universidade Norueguesa de Ciências da Vida, Calvin Wan e Geoffrey Qiping Shen, da Universidade Politécnica de Hong Kong, na China, e Hongyun Han do Centro de Desenvolvimento Agrícola e Rural da Universidade Hangzow, na China. Quanto a co-autoria na produção, dois desses autores produziram dois artigos em co-autoria, sendo eles Calvin Wan e Geoffrey Qiping Shen (figura 2).

Tabela 2: Autores com mais artigos publicados.

\begin{tabular}{|c|c|c|}
\hline Autores & Frequência & Afiliação \\
\hline Katrine Soma & 2 & Universidade Norueguesa de Ciências da Vida \\
\hline Calvin Wan & 2 & Universidade Politécnica de Hong Kong, na China \\
\hline Geoffrey Qiping Shen & 2 & Universidade Politécnica de Hong Kong, na China \\
\hline Hongyun Han & 2 & Universidade Hangzow, na China \\
\hline
\end{tabular}

A figura 2 foi gerada pelo VOSViewer ${ }^{\circledR}$ e representa o mapa dos autores com mais produções e com coautoria. O mapa é gerado pelo software através do arquivo no formato (.txt) com os dados bibliográficos dos 56 artigos pesquisado na WOS. As diferentes cores dos itens indicam os clusters às quais pertence cada autor. Neste caso, os clusters representam a similaridade de domínio de estudo entre os autores.

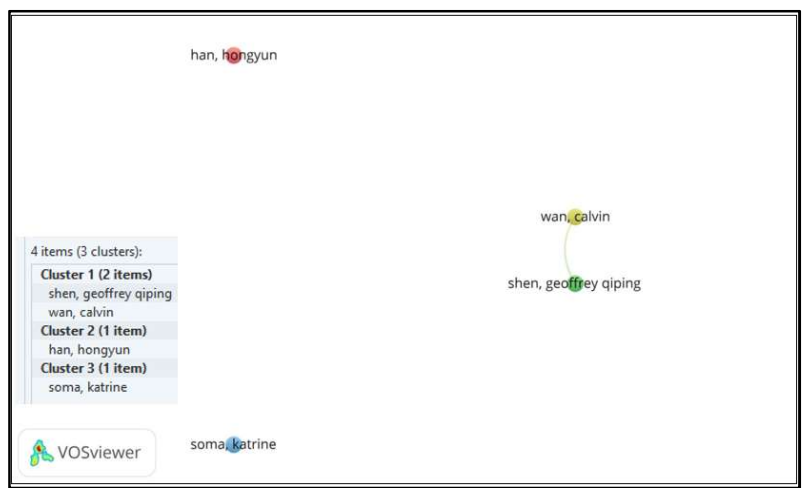

Figura 2: Mapa dos autores com mais produções e com coautoria.

Nessa análise identificou-se a formação de 3 clusters de autores, o primeiro cluster formado pelos professores Calvin Wan e Geoffrey Qiping Shen, o segundo por Hongyun Han e o terceiro por Katrine Soma. 
Sendo o cluster 2 e 3 sem coautoria e cada um com dois trabalhos, no caso os professores Hongyun Han e Katrine Soma, e o cluster 1 que apresenta co-autoria entre os professores Calvin Wan e Geoffrey Qiping Shen, sendo que cada um além da coautoria apresentam 2 trabalhos.

Quanto ao conteúdo dos clusters, o cluster 1 formado por autores Calvin Wan e Geoffrey Qiping Shen que publicaram em coautoria, apresentam seus artigos referentes à participação social na gestão ambiental e no gerenciamento de resíduos sólidos. Tratam dos determinantes da disposição para apoiar medidas políticas sobre reciclagem, destacando que entender o que contribui para o apoio público na reciclagem é uma tarefa fundamental para as decisões políticas. (WAN et al., 2015; WAN et al., 2017).

No cluster 2, Hongyun Han aborda a temática do gerenciamento de recursos hídricos, destacando que a falta de participação pública na Avaliação de Impacto Ambiental é uma das razões fundamentais para a deterioração dos ambientes ribeirinhos e superexploração de água, bem como os desafios dos tomadores de decisão na redução de conflitos entre o aumento da demanda de alimentos e a redução do suprimento de água sem prejudicar progresso da urbanização e do setor industrial (HAN, 2018). O outro artigo trata sobre resíduos sólidos, ressaltando a necessidade de facilitar a participação pública na separação da fonte geradora de resíduos pela adoção de políticas mistas que combinam o voluntário com o obrigatório bem como abordagens de educação (HAN, 2017).

No cluster 3, formado por Katrine Soma aborda a participação na gestão de áreas protegidas, nesse caso áreas de zonas costeiras, destacam que a participação das partes interessadas pode contribuir para melhorar as decisões complexas de gestão ambiental. Bem como o desafio de lidar com a pluralidade existente nos tipos de valores sociais que as pessoas têm quando decidem sobre o futuro tratamento de recursos ambientais que são compartilhados entre si (SOMA et al., 2010).

\section{Temas abordados nas produções}

Os artigos abordam 5 áreas temáticas em relação ao foco principal da participação social, sendo $52 \%$ dos artigos, tratando da participação social na gestão de Resíduos Sólidos, evidenciando o grande desafio enfrentado pelos países diante dessa problemática. Em segundo lugar, aparecem à gestão de Recursos Hídricos e a Gestão Ambiental e Sociedade com 18\% dos artigos respectivamente. Em terceiro lugar aparecem as Áreas Protegidas presente em 9\% e, por último, Mudanças Climáticas e Energias Renováveis somente em $3 \%$, como mostra o gráfico 2 abaixo. 0 tema que mais cresceu dentro das produções científicas analisadas considerando o ano de 2017 que teve 16 artigos publicados com a temática pesquisada, foi em relação à participação social voltada para a problemática ambiental dos resíduos sólidos em nível mundial.

No caso de Ferreira et al. (2017) os autores realizaram uma pesquisa de estudo de caso em Belo Horizonte no estado de Minas Gerais no Brasil, quanto à gestão de resíduos sólidos e afirmam que a construção de soluções de gestão sustentável, especialmente os problemas de gestão de resíduos em geral, deve-se focar na participação pública da população, nas tecnologias e na investigação sobre a melhor alternativa para a cidade. Na tabela 1, é possível visualizar os autores que publicaram em cada uma das áreas temáticas que foram identificadas. 


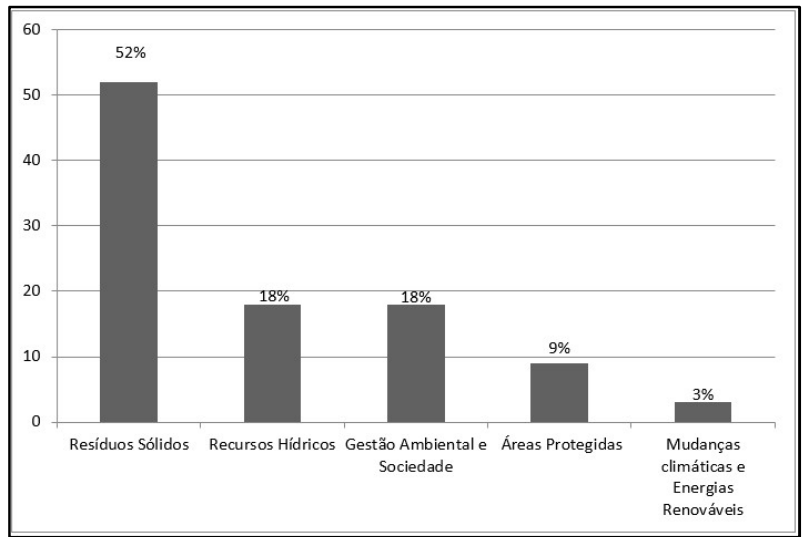

Gráfico 2: Porcentagem dos temas abordados nas produções científicas.

Tabela 1: Autores por áreas temáticas.

\begin{tabular}{|c|c|}
\hline Autores & Área temática \\
\hline $\begin{array}{l}\text { Gutberlet (2008); Agamuthu et al. (2011); Ezebilo et al. (2011); Che et al. (2013); Fernandes et al. (2013); Rousta et al. } \\
\text { (2013); Cimmuto et al. (2014); Vasileva et al. (2014); Wan et al. (2015); Hu et al. (2015); Ma et al. (2016); Agovino et al. } \\
\text { (2016); Zhang et al. (2016); Triguero et al. (2016); Ferreira et al. (2017); Han et al. (2017); Huici et al. (2017); Garnett et al. } \\
\text { (2017); Xiao et al. (2017); Loan et al. (2017); Majeed et al. (2017); Linzalone et al. (2017); Raharjo et al. (2017); Sisto et al. } \\
\text { (2017); Chen et al. (2018); Manomaivibool et al. (2018); Tovar (2018); Trimurni et al. (2018); Meng et al. (2018). }\end{array}$ & Resíduos Sólidos. \\
\hline $\begin{array}{l}\text { Fleeger et al. (2008); Wilson et al. (2008); Costa et al. (2009); Bergsma et al. (2012); Hernández et al. (2014); Siegmund- } \\
\text { Schultze et al. (2015); Simpson et al. (2015); Frascari et al. (2018); Han et al. (2018); Kendy et al. (2018). }\end{array}$ & Recursos Hídricos. \\
\hline $\begin{array}{l}\text { Mertens et al. (2011); Giaretta et al. (2012); Pedde et al. (2015); Morais et al. (2016); Weddfelt et al. (2016); Bai et al. } \\
\text { (2017); Locastro et al. (2017); Mishenina et al. (2017); Rubio et al. (2017); Wan et al. (2017); }\end{array}$ & $\begin{array}{l}\text { Gestão Ambiental } \\
\text { e Sociedade. }\end{array}$ \\
\hline Soma et al. (2010); Soma et al. (2010); Jansujwicz et al. (2013); Cardoso et al. (2015); Ceratti et al. (2016). & Áreas Protegidas \\
\hline Marengo et al (2017); Konti at al (2018). & $\begin{array}{l}\text { Mudanças } \\
\text { Climáticas e } \\
\text { Energias } \\
\text { Renováveis. }\end{array}$ \\
\hline
\end{tabular}

Em primeiro lugar com o destaque para a questão dos resíduos sólidos, Rousta et al. (2013) discute a melhoria da participação dos habitantes no sistema de gestão de resíduos, apontando a necessidade de mudanças em fatos como a conveniência e fácil acesso às estações de reciclagem nos sistemas locais de gestão de RSU, e revisão da regulamentação e política atuais.

A participação social é tratada como uma necessidade e instrumento para efetividade das políticas públicas de gerenciamento de resíduos sólidos (GUTBERLET, 2008; AGAMUTHU et al., 2011; EZEBILO et al., 2011). Atitudes positivas individuais em relação ao gerenciamento de resíduos sólidos foram constatadas como respostas pressionadas pelo contexto social a fim de seguir a linha de força moral referente à preservação do meio ambiente (FERNANDES, 2013). Quanto aos instrumentos de participação social nessa área, foram apontados: a adoção de um esquema porta-a-porta apoiado por um sistema de informação e educação para os cidadãos, assim como campanhas de comunicação relacionadas à prestação de informações sobre os benefícios da reciclagem para aumentar o interesse e melhoria dos resultados da separação e recolhimento de resíduos (CIMMUTO, 2014; VASILEVA, 2014).

Em segundo lugar estão as temáticas de recursos hídricos e a gestão ambiental e sociedade. No que tange aos recursos hídricos, a Participação Social apresenta-se como um princípio norteador de políticas públicas, destacando o papel das mulheres nessa participação especialmente na tarefa da gestão e consumo da água (WILSON, 2008; HERNÁNDEZ, 2014).

A melhoria das condições de participação, especialmente nos comitês, constitui um avanço democrático na construção de governança (COSTA et al., 2009). Porém, Giaretta et al. (2012) chama atenção 
para a necessidade de debate sobre o real funcionamento democrático de tais instituições e seus resultados enquanto locais de debates e promoção de troca de conhecimentos entre sociedade e governo local.

Na temática da Gestão Ambiental e Sociedade a participação social é tratada como um desafio tendo em vista uma cultura cívica restrita, que abre oportunidades para, entre outras coisas, o clientelismo, uma visão de curto prazo e ações que favoreçam interesses individuais em vez de interesses coletivos. Uma sociedade em que a cultura cívica é subdesenvolvida, a responsabilidade pelos problemas, neste caso os problemas ambientais, recai sobre os "outros". Governo, empresários e cidadãos culpam-se mutuamente sem, no entanto, serem capazes de construir alternativas viáveis e racionais para o bem geral (PEDDE et al., 2015).

Nos seus estudos sobre a participação social, em relação à gestão de áreas de proteção ambiental em municípios na Noruega, Soma et al. (2010) afirma que a formulação de políticas ambientais pode ser desafiadora por causa da pressão de fortes interesses. Isso resulta em menos consideração sobre o que é melhor para a comunidade em geral. A participação das partes interessadas pode contribuir para melhorar as decisões complexas de gestão ambiental. É um desafio lidar com a pluralidade existente de tipos de valores sociais que as pessoas têm quando decidem sobre o futuro tratamento de recursos ambientais que são compartilhados entre as pessoas (SOMA et al., 2010).

Marengo et al. (2017) após desenvolver sua pesquisa em Santos, no Brasil, apontam que estratégias para a compreensão e conscientização pública dos efeitos tangíveis das mudanças climáticas são fundamentais para o desenvolvimento de opções políticas. Nessa pesquisa uma equipe multidisciplinar e multinacional de cientistas naturais e sociais dos EUA, Reino Unido e Brasil desenvolveram um projeto chamado METROPOLE, que desenvolveu uma abordagem participativa onde atores públicos se envolvem plenamente na definição do problema de pesquisa e na avaliação dos resultados.

A importância da participação social é também reforçada por Konti at al. (2018) ao pesquisarem sobre a produção de energias renováveis, visto que a essa questão tornou-se uma prioridade na União Europeia, dado o esgotamento dos recursos fósseis, combustíveis e a deterioração do ambiente. Segundo Konti at al. (2018), os resíduos e especificamente resíduos biológicos, a fração orgânica de resíduos sólidos, são considerados como matéria-prima ideal para a produção de bioetanol. No entanto, a produção do bioetanol através de resíduos biológicos em grande escala é um projeto complexo que requer a participação e o engajamento das diferentes partes interessadas envolvidas nas diferentes etapas do processo, desde a coleta dos resíduos até a produção do produto e a gestão dos resíduos.

\section{CONSIDERAÇÕES FINAIS}

A investigação da produção científica acerca da participação social na gestão ambiental municipal mostrou-se como campo de estudo em relativo crescimento, a partir de 2015, porém, ainda de forma fragmentada e sem redes de coautoria entre instituições e países. Verificou-se, uma pequena concentração das publicações no Journal of Cleaner Production e o Resources e o Conservation and Recycling, com 5 artigos publicados em cada um desses periódicos, bem como na China com 8 artigos, seguido da Noruega e do Brasil 
com 4 respectivamente.

As análises dos dados bibliográficos permitiram verificar que, além de recentes, estas pesquisas têm se concentrado na avaliação e desenvolvimento dessa participação em questões e áreas temáticas como: quanto à problemática dos resíduos sólidos, a questão dos recursos hídricos, da gestão de áreas protegidas, do desenvolvimento da gestão ambiental e a sociedade, e uma pequena presença quanto às mudanças climáticas e energias renováveis. O tema que mais cresceu dentro das produções científicas analisadas, foi em relação à participação social voltada para a problemática ambiental dos resíduos sólidos, evidenciando a mesma como um problema gigantesco e em nível mundial. Parece haver um consenso sobre a importância do papel da participação social na gestão ambiental municipal, porém apresentam-se poucos estudos. Em pesquisas futuras podem ser ampliadas as investigações para outros bancos de dados, como o Scopus.

\section{REFERÊNCIAS}

AGAMUTHU, P.; FAUZIAH, S. H.. Challenges and issues in moving towards sustainable landfilling in a transitory country-Malaysia. Waste Management \& Research, v.29, n.1, p.13-19, 2011. DOI: http://doi.org://10.1177/0734242X10383080

AGOVINO, M.; GAROFALO, A.; MARIANI, A.. Effects of environmental regulation on separate waste collection dynamics: empirical evidence from Italy. Journal of Cleaner Production, v.124, 2016. DOI:

http://doi.org/10.1016/i.jclepro.2016.02.082

ÁVILA, R. D.; MALHEIROS, T. F.. O Sistema Municipal de Meio Ambiente no Brasil: avanços e desafios. Revista Saúde e Sociedade, São Paulo, v.21, p.33-47, 2012.

AVRITZER, L.; SOUZA, C. H. L.. Conferências nacionais: atores, dinâmicas participativas e efetividades. Brasília: IPEA, 2013.

BAI, X.; MCPHEARSON, T.; CLEUGH, H.; NAGENDRA, H.; TONG, X.; ZHU, T.; ZHU, Y. G.. Linking Urbanization and the Environment: Conceptual and Empirical Advances. Annu. Aannual Review of Environmental and Resources, v.42, p.215-240, 2017. DOI: https://doi.org/10.1146/annurevenviron-102016-061128

BERGSMA, E.; GUPTA, J.; JONG, P.. Does individual responsibility increase the adaptive capacity of society? The case of local water management in the Netherlands. Resources, Conservation and Recycling, v.64, p.13-22, 2012. DOI: http://doi.org/10.1016/j.resconrec.2012.03.006

BRASIL. Constituição da República Federativa do Brasil de 5 de outubro de 1988. Brasília: DOU, 1988.

BURSZTYN, M.; BURSZTYN, M. A.. Fundamentos de política e gestão ambiental: os caminhos do desenvolvimento sustentável. Rio de Janeiro: Garamond, 2012.

CARDOSO, S. L. C.; SOBRINHO, M. V.; VASCONCELLOS, A. M. A.. Gestão ambiental de parques urbanos: o caso do Parque Ecológico do Município de Belém Gunnar Vingren. Revista Brasileira de Gestão Urbana, v.7, n.1, p.74-90, 2015.

CARVALHO, M. C.. Participação social no Brasil hoje. São
Paulo: Instituto Polis, 1998.

CERATI, T. M.; SOUZA, A. Q.. Participación social en la gestión ambiental: estudio de caso en una unidad de conservación urbana en el municipio de São Paulo, Brasil. Estudios Demográficos y Urbanos, v.31, n.1, p.87-113, 2016.

CHE, Y.; YANG, K.; JIN, Y.; ZHANG, W.; SHANG, Z.; TAI, J.. Residents' concerns and attitudes toward a municipal solid waste landfill: integrating a questionnaire survey and GIS techniques. Environ Monit Assess, v.185, n.12, 2013. DOI: http://doi.org/10.1007/s10661-013-3308-y

CHEN, F.; CHEN, H.; GUO, D.; HAN, S.; LONG, R.. How to achieve a cooperative mechanism of MSW source separation among individuals $\mathrm{d} A \mathrm{n}$ analysis based on evolutionary game theory. Journal of Cleaner Production, v.195, 2018. DOI: https://doi.org/10.1016/j.jclepro.2018.05.226

CIMMUTO, A. D.; MANNOCCI, A.; RIBATTI, D.; BOCCIA, A.; TORRE, G.. Impact on knowledge and behaviour of the general population of two diferente methods of solid waste management: An explorative cross-sectional study. Waste Management \& Research, v.32, n.6, p.556-561, 2014. DOI: https://doi.org://10.1177/0734242X14536461

COSTA, G. M.; COSTA, H. S. M.; DIAS, J. B.; WELTER, M. G.. The role of municipal committees in the development of an integrated urban water policy in Belo Horizonte, Brazil. Water Science \& Technology, v.60, n.12, 2009. DOI: https://doi.org://10.2166/wst.2009.742

EZEBILO, E. E.; ANIMASAUN, E. D.. Households' perceptions of private sector municipal solid waste management services: A binary choice analysis. International Journal of Environmental Science and Technology, v.8, n.4, p.677-686, 2011.

FARIAS, S. C. G. O SIMMA e a articulação da gestão ambiental nos municípios brasileiros: o caso de Rio das Ostras-RJ. Revista Ambiente \& Educação, v.16, n.2, 2011.

FERNANDES, L. G.; SANSOLO, D. G.. Percepção ambiental dos moradores da cidade de São Vicente sobre os resíduos sólidos na Praia do Gonzaguinha, SP, Brasil. Revista da Gestão Costeira Integrada, v.13, n.3, p.379-389, p.2013. 
FERNANDES, V.; SAMPAIO, C. A. C.. Problemática ambiental ou problemática socioambiental?. Desenvolvimento e Meio Ambiente, Curitiba, n.18, p.87-94, 2008.

FERREIRA, E. M.; BARROS, R. T. V.; SOVIAR, J.. Brazilian waste management: Belo Horizonte's case study of sustainable management. Procedia Engineering, v.192, p.171-176, 2017. DOI: https://doi.org://10.1016/j.proeng.2017.06.030

FLEEGER, W. E.; BECKE, M. L.. Creating and sustaining community capacity for ecosystem-based management: Is local government the key?. Journal of Environmental Management, v.88, p.1396-1405, 2008. DOI: http://doi.org/10.1016/i.jenvman.2007.07.018

FRASCARI, D.; ZANAROLI, G.; MOTALEB, M. A.; ANNEN, G.; BELGUITH, K.; BORIN, S.; CHOUKR-ALLAH, R.; GIBERT, C.; JAOUANI, A.; KALOGERAKIS, N.; KARAJEH, F.; RAULT, P. A. K.; KHADRA, R.; KYRIACOU, S.; LI, W.; MOLLE, B.; MULDER, M.; OERTL, E.; ORTEGA, C. V.. Integrated Technological and Management Solutions for Wastewater Treatment and Efficient Agricultural Reuse in Egypt, Morocco, and Tunisia. Integrated Environmental Assessment and Management, v.9999, p.1-16, 2018. DOI:

http://doi.org/10.1002/ieam.4045

GARBELINE, C. B.. Reflexão sobre participação social: barreiras e estratégias. Geosul, Florianópolis, v.32, n.64, p.165-178, 2017.

GARNETT, K.; COOPER, T.; LONGHURST, P.; JUDE, S.; TYRREL, S.. A conceptual framework for negotiating public involvement in municipal waste management decisionmaking in the UK. Waste Management, v.66, p.210-221, 2017. DOI: http://doi.org/10.1016/j.wasman.2017.04.022

GIARETTA, J. B. Z.; FERNANDES, V.; PHILIPPI JUNIOR, A.. Desafios e condicionantes da participação social na gestão ambiental municipal no Brasil. O\&S, Salvador, v.19, n.62, p.527-548, 2012.

GUTBERLET, J.. Empowering collective recycling initiatives: Video documentation and action research with a recycling co-op in Brazil Resources, Conservation and Recycling, v.52, p.659-670, 2008. DOI: http://doi.org/10.1016/j.resconrec.2007.08.006

HAN, H.; XIA, S.; JIANG, Y.. Challenging Issues over Sustainable Water Management in Coastal Area from China. Journal of Coastal Research, v.83, p.946-958, 2018. DOI: https://doi.org/10.2112/SI83-157.1

HAN, H.; ZHANG, Z.. The impact of the policy of municipal solid waste source-separated collection on waste reduction: a case study of China. Journal of Material Cycles Waste Management, v.19, p.382-393, 2017. DOI: http://doi.org/10.1007/s10163-015-0434-3

HERNÁNDEZ, R. V.; DOMÍNGUEZ, D. L.. Think of water from a social perspective. A research project in Havana. Procedia: Social and Behavioral Sciences, v.132, p.473-478, 2014. DOI: http://doi.org/10.1016/j.sbspro.2014.04

HU, H.; LI, X.; NGUYEN, A. D.; KAVAN, P.. A Critical Evaluation of Waste Incineration Plants in Wuhan (China) Based on Site Selection, Environmental Influence, Public Health and Public
Participation. International Journal of Environmental

Research and Public Health, v.12, p.7593-7614, 2015. DOI: http://doi.org/10.3390/ijerph120707593

HUIC, O.; SKOVGAARD, M.; CONDARCO, G.; JØRS, E.; JENSEN, O. C.. Management of Empty Pesticide Containers: A Study of Practices in Santa Cruz, Bolivia. Environmental Health Insights, v.11, p.1-7, 2017. DOI: https://doi.org/10.1177/1178630217716917

JANSUJWICZ, J. S. CALHOUN, A. J. K.; LILIEHOLM, R. J.. The Maine Vernal Pool Mapping and Assessment Program: Engaging Municipal Officials and Private Landowners in Community-Based Citizen Science. Environmental Management, v.52, p.1369-1385, 2013. DOI: http://doi.org/10.1007/s00267-013-0168-8

KENDY, E.; AYLWARD, B.; ZIEMER, L. S.; RICHTER, B. D.; COLBY, B. G.; GRANTHAM, T. E.; SANCHEZ, L.; DICHARRY, W. B.; POWELL, E. M.; MARTIN, S.; CULP, P. W.; SZEPTYCKI, L. F.; KAPPE, C. V.. Water transactions for streamflow restoration, water supply reliability, and rural economic vitality in the western united states. Journal of the American Water Resources Association, v.54, n.2, 2018.

KONTI, A.; DAMIGOS, D.. Exploring strengths and weaknesses of bioethanol production from biowaste in Greece using Fuzzy Cognitive Maps. Energy Policy, v.112, p.4-11, 2018. DOI: http://doi.org/10.1016/i.enpol.2017.09.053

LILLO, F. G.; CORTES, E. C.; LAJARA, B. M.; GARCIA, M. U. Mapping the Intellectual Structure of Research on 'Born Global' Firms and INVs: A Citation/Cocitation Analysis. Management International Review, v.57, p.631-652, 2017. DOI: http://doi.org/10.1007/s11575-016-0308-5

LINZALONE, N.; COI, A.; LAURIOLA, P.; LUISE, D.; PEDONE, A.; ROMIZI, R.; SALLESE, D.; BIANCHI, F.. Participatory health impact assessment used to support decision-making in waste management planning: A replicable experience from Italy. Waste Management, v.59, p.557-566, 2017. DOI: http://doi.org/10.1016/j.wasman.2016.09.035

LOAN, L. T. T.; NOMURA, H.; TAKAHASHI, Y.; YABE, M.. Psychological driving forces behind households' behaviors toward municipal organic waste separation at source in Vietnam: a structural equation modeling approach. Journal of Material Cycles and Waste Management, v.19, p.10521060, 2017. DOI: http://doi.org10.1007/s10163-017-0587-3

LOCASTRO, J. K.; MIOTTO, J. L.; ANGELIS, B. L. D.; CAXAMBU, M. G.. Avaliação do uso sustentável da arborização urbana no município de Cafeara, Paraná. Ciência Florestal, Santa Maria, v.27, n.2, p.549-556, 2017.

LÓPEZ, O. C.. Procedimiento metodológico para ejecutar el proceso de ordenamiento ambiental en Cuba. Mercator, Fortaleza, v.13, n.2, p.209-226, 2014. DOI: http://doi.org/10.4215/RM2014.1302

MA, J.; HIPEL, K. W.. Exploring social dimensions of municipal solid waste management around the globe - A systematic literature review. Waste Management, v.56, p.3-12, 2016. DOI: http://doi.org/10.1016/i.wasman.2016.06.041

MAJEED, A.; BATOOL, S. A.; CHAUDHRY, M. N.. Informal 
Waste Management in the Developing World: Economic Contribution Through Integration With the Formal Sector. Waste Biomass Valor, v.8, p.679-694, 2017. DOI: http://doi.org/10.1007/s12649-016-9648-4

MANOMAIVIBOOL, P.; SRIVICHAI, M.; UNROJ, P.; DOKMAINGAM, P.. Chiang Rai Zero Waste: Participatory action research to promote source separation in rural areas. Resources, Conservation \& Recycling, v.136, p.142-152, 2018. DOI: https://doi.org/10.1016/i.resconrec.2018.04.002

MARENGO, J. A.; NUNES, L. H.; SOUZA, C. R. G.; HARARI, J.; KARGER, F. M.; GRECO, R.; HOSOKAWA, E. K.; TABUCHI, E. K.; MERRILL, S. B.; REYNOLDS, C. J.; PELLING, M.; ALVES, L. M.; ARAGÃO, L. E.; CHOU, S. C.; MOREIRA, F.; PATERSON, S.; LOCKMAN, J. T.; GRAY, A. G.. A globally deployable strategy for co-development of adaptation preferences to sea-level rise: the public participation case of Santos, Brazil. Nat Hazards, v.88, p.39-53, 2017. DOI: http://doi.org/10.1007/s11069-017-2855-x

MEDEIROS, G. A.; GIORDANO, L. C.; REIS, F. A. G. V.. Gestão Ambiental. In: ROSA, A. H.; FRACETO, L. F.; MOSCHINICARLOS, V.. Meio Ambiente e Sustentabilidade. Porto Alegre: Bookman, 2012. p.375-406.

MENG, X.; WEN, Z.; QIAN, Y.. Multi-agent based simulation for household solid waste recycling behavior. Resources, Conservation and Recycling, v.128, p.535-545, 2018. DOI: http://doi.org/10.1016/i.resconrec.2016.09.033

MERTENS, F.; TÁVORA, R.; FONSECA, I. F.; GRANDO, R.; CASTRO, M.; DEMEDA, K.. Redes sociais, capital social e governança ambiental no Território Portal da Amazônia. Acta Amazônica, v.41, n.4, p.481-492, 2011.

MISHENINA, N. V.; YAROVA, I. Y. E.; MISHENINA, H. A.. Desenvolvimento de mecanismos de parceria públicoprivada no âmbito da gestão da natureza em condições de descentralização. Marketing and management of innovations, Varsóvia, 2017.

MORAIS, V. S.; CHAVES, A. P. L. Percepção dos gestores municipais de saúde relacionada à saúde ambiental: consórcio intermunicipal de saúde Cerrado Tocantins Araguaia. Saúde Social, São Paulo, v.25, n.2, p.349-360, 2016. DOI: http://doi.org/10.1590/S0104-12902016149984

PEDDE, V.; FIGUEIREDO, J. A. S.; NUNES, M.F.; PRODANOV, C. C.. Environment and society: the Sinos River Basin and public policies. Brazilian Journal of Biology, v.75, n.2, p.128136, 2015. DOI: http://doi.org/10.1590/1519-6984.1313

PEREIRA, D. E. S.. O dever de participação na proteção em matéria ambiental na legislação brasileira. Direito e Política, Itajaí, v.10, n.1, 2015.

RAHARJO, S.; MATSUMOTO, T.; IHSAN, T.; RACHMAN, I.; GUSTIN, L.. Community-based solid waste bank program for municipal solid waste management improvement in Indonesia: a case study of Padang city. Journal of Material Cycles and Waste Management, v.19, p.201-212, 2017. DOI: http://doi.org/10.1007/s10163-015-0401-z

ROUSTA, K.; EKSTRÖM, K. M.. Assessing Incorrect Household Waste Sorting in a Medium-Sized Swedish City.

Sustainability, v.5, p.4349-4361, 2013. DOI: http://doi.org/10.3390/su5104349

RUBIO, M. C.; RUBIO, C.; SALOMÓN, S.; ABRAHAM, E.. Conservation of ecosystem services in high-altitude Andean wetlands: social participation in the creation of a natural protected área. Ecología Austral, v.27, p.177-192, 2017. DOI: http://doi.org/10.25260/EA.17.27.1.1.271

SIEGMUND-SCHULTZE, M.; RODORFF, V.; KOPPEL, J.; SOBRAL, M. C. Paternalism or participatory governance? Efforts and obstacles in implementing the Brazilian water policy in a large watershed. Land Use Policy, v.48, p.120130, 2015. DOI:

http://doi.org/10.1016/j.landusepol.2015.05.024

SIMÕES, G. L.; SIMÕES, J. M.. Uma análise sobre a participação social nos projetos sociais da FIOCRUZ. In: FÓRUM DA PÓS-GRADUAÇÃO DA UFRRJ, 10. Anais. SEROPÉDICA. Anais. Rio de Janeiro: UFRRJ, 2015.

SIMPSON, H.; LÖE, R.; ANDREY, J.. Vernacular knowledge and water management: Towards the integration of expert science and local knowledge in Ontario, Canada. Water Alternatives, v.8, n.3, p.352-372, 2015.

SIQUEIRA, L. C. Política ambiental para quem?. Ambiente \& Sociedade, Campinas, v.11, n.2, p.425-437, 2008.

SISTO, R.; SICA, E.; LOMBARDI, M.; PROSPERI, M.. Organic fraction of municipal solid waste valorisation in Southern Italy: the stakeholders' contribution to a long-term strategy definition. Journal of Cleaner Production, v.168, 2017. DOI: http://doi.org/10.1016/j.jclepro.2017.08.186

SOMA, K.; NORWAY, Å.. Framing Participation with Multicriterion Evaluations to Support the Management of Complex Environmental Issues. Environmental Policy and Governance, v.20, p.89-106, 2010. DOI: http://doi.org/10.1002/eet.534

SOMA, K.; VATN, A.. Is There Anything Like a Citizen? A Descriptive Analysis of Instituting a Citizen's Role to Represent Social Values at the Municipal Level. Environmental Policy and Governance, v.20, p.30-43, 2010. DOI: $\underline{\text { http://doi.org/10.1002/eet.529 }}$

TOVAR, L. F.. Formalización de las organizaciones de recicladores de oficio en Bogotá: reflexiones desde la economía popular. Íconos. Revista de Ciencias Sociales, Quito, n.62, p.39-63, 2018. DOI:

http://doi.org/10.17141/iconos.62.2018.3230

TRIGUERO, A.; ALVAREZ-ALEDO, C.; CUERVA, M. C.. Factors influencing willingness to accept different waste management policies: empirical evidence from the European Union. Journal of Cleaner Production, v.138, 2016. DOI: http://doi.org/10.1016/i.jclepro.2016.05.119

TRIMURNI, F. D.. The participation of community-based organizations on waste management in the city municipal of Medan. IOP Conf. Series: Earth and Environmental Science, v.126, 2018. DOI: http://doi.org/10.1088/1755$1315 / 126 / 1 / 012141$

VALDIVIES O. P.. Facilitadores institucionales y sociales para la gobernanza local de los riesgos medioambientales: Análisis empírico con municipios chilenos. Opinião Pública, 
Campinas, v.23, n.3, 2017.

VASILEVA, E.; IVANOVA, D.. Towards a sustainable consumer model: the case study of Bulgarian recyclers. International Journal of Consumer Studies, v.38, p.475-484, 2014. DOI: http://doi.org/10.1111/ijcs.12123

WAN, C.; SHEN, G. Q.; CHOI, S.. A review on political factors influencing public support for urban environmental policy. Environmental Science and Policy, v.75, p.70-80, 2017. DOI: http://doi.org/10.1016/j.envsci.2017.05.005

WAN, C.; SHEN, G. Q.; YU, A.. Key determinants of willingness to support policy measures on recycling: A case study in Hong Kong. Environmental Science \& Policy, v.54, p.409-418, 2015. DOI:

http://doi.org/10.1016/j.envsci.2015.06.023

WEDDFELT, E.; VACCARI, M.; TUDOR, T.. The development of environmental visions and strategies at the municipal level:
Case studies from the county of Ostergotland in Sweden. Journal of Environmental Management, v.179, 2016. DOI: http://doi.org/10.1016/i.jenvman.2016.04.050

WILSON, Z.; MALAKOANA, M.; GOUNDEN, T.. Trusting consumers: Involving communities in municipal water service decision making in Durban, South Africa. Water SA, v.34, n.2, 2008

XIAO, L.; ZHANG, G.; ZHU, Y.; LIN, T.. Promoting public participation in household waste management: A survey based method and case study in Xiamen city, China. Journal of Cleaner Production v.144, 2017. DOI: http://doi.org/10.1016/j.jclepro.2017.01.022

ZHANG, S.; ZHANG, M.; YU, X.; REN, H.. What keeps Chinese from recycling: Accessibility of recyclingfacilities and the behavior. Resources, Conservation and Recycling, v.109, p.176-186, 2016.

http://doi.org/10.1016/j.resconrec.2016.02.008

A CBPC - Companhia Brasileira de Produção Científica (CNPJ: 11.221.422/0001-03) detém os direitos materiais desta publicação. Os direitos referem-se à publicação do trabalho em qualquer parte do mundo, incluindo os direitos às renovações, expansões e disseminações da contribuição, bem como outros direitos subsidiários. Todos os trabalhos publicados eletronicamente poderão posteriormente ser publicados em coletâneas impressas sob coordenação da Sustenere Publishing, da Companhia Brasileira de Produção Científica e seus parceiros autorizados. Os (as) autores (as) preservam os direitos autorais, mas não têm permissão para a publicação da contribuição em outro meio, impresso ou digital, em português ou em tradução. 